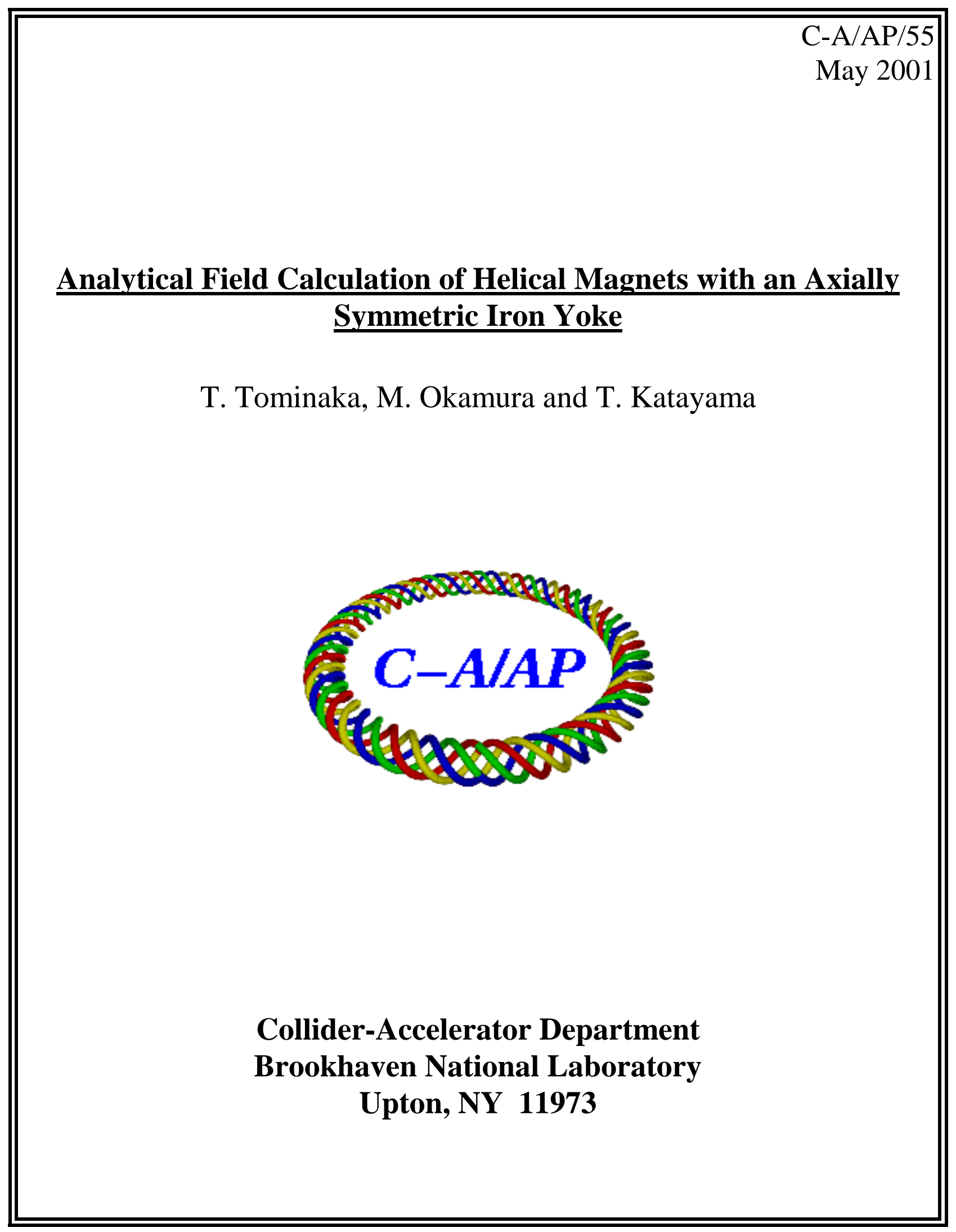




\title{
Analytical Field Calculation of Helical Magnets \\ with an Axially Symmetric Iron Yoke
}

\author{
T. Tominaka ${ }^{\mathrm{a}}$, M. Okamura ${ }^{\mathrm{a}}$, and T. Katayama, \\ aRIKEN (The Institute of Physical and Chemical Research), \\ 2-1, Hirosawa, Wako, Saitama, 351-0198, Japan \\ ${ }^{\mathrm{b}}$ Center for Nuclear Study, School of Science, University of Tokyo, \\ RIKEN, 2-1, Hirosawa, Wako, Saitama, 351-0198, Japan
}

Abstract

The magnetic field due to a current flowing in a helical conductor placed inside a cylindrical hole in iron is investigated. In order to calculate the contribution of an axially symmetric iron yoke on helical magnets, the 3-dimensional potential problem is solved. The obtained results are applied for the helical dipole magnet for RHIC.

\section{INTRODUCTION}

The contribution of an axially symmetric iron yoke on the helical magnets has been examined by Caspi [1]. On this potential problem, the boundary condition on the inner surface of iron set by Caspi seems wrong. This potential problem is solved with the corrected boundary condition.

\section{FIELD OF A SINGLE HELICAL CURRENT CONDUCTOR}

In this paper, the magnetic induction $\mathrm{B}$, the magnetic scalar potential $\phi_{\mathrm{m}}$ and the vector potential A are defined as follows,

$$
B=-\mu \nabla \phi_{m}=\nabla \times A
$$

Similarly, the relation between the magnetic induction B, and the magnetic field intensity $\mathrm{H}$ is defined as follows,

$B=\mu_{0}(H+M)=\mu_{0}\left(1+\chi_{m}\right) H=\mu_{0} \kappa_{m} H=\mu H$

where $\mu$ is the absolute permeability, $\chi_{\mathrm{m}}$ is the magnetic susceptibility, and $\kappa_{\mathrm{m}}$ is the relative permeability. 
On the case that a single helical current carrying conductor with a pitch length $\mathrm{L}$ (= $2 \pi / \mathrm{k})$ is located at some point $(\mathrm{r}=\mathrm{b}, \theta=\varphi)$ at the $\mathrm{z}=0$ plane, as shown in Figs. 1 and 2 , the magnetic scalar potential $\phi_{\mathrm{m}}$ and the field B due to a single helical current carrying conductor are written in the following forms, [2]

for $r<b$,

$\phi_{m}(r, \theta, z)=-\frac{I}{2 \pi} k z-\frac{I}{\pi} k b \sum_{n=1}^{\infty} K_{n}^{\prime}(n k b) I_{n}(n k r) \sin [n(\theta-\varphi-k z)]$

$\left\{\begin{array}{l}B_{r}(r, \theta, z)=\frac{\mu_{0} I}{\pi} k^{2} b \sum_{n=1}^{\infty} n K_{n}^{\prime}(n k b) I_{n}^{\prime}(n k r) \sin [n(\theta-\varphi-k z)] \\ B_{\theta}(r, \theta, z)=\frac{\mu_{0} I}{\pi} k b \sum_{n=1}^{\infty} n K_{n}^{\prime}(n k b) \frac{I_{n}(n k r)}{r} \cos [n(\theta-\varphi-k z)] \\ B_{z}(r, \theta, z)=\frac{\mu_{0} I}{2 \pi} k-\frac{\mu_{0} I}{\pi} k^{2} b \sum_{n=1}^{\infty} n K_{n}^{\prime}(n k b) I_{n}(n k r) \cos [n(\theta-\varphi-k z)]\end{array}\right.$

Similarly, for $r>b$,

$$
\begin{aligned}
& \phi_{m}(r, \theta, z)=-\frac{I}{2 \pi} \theta-\frac{I}{\pi} k b \sum_{n=1}^{\infty} I_{n}^{\prime}(n k b) K_{n}(n k r) \sin [n(\theta-\varphi-k z)] \\
& \left\{\begin{array}{l}
B_{r}(r, \theta, z)=\frac{\mu_{0} I}{\pi} k^{2} b \sum_{n=1}^{\infty} n I_{n}^{\prime}(n k b) K_{n}^{\prime}(n k r) \sin [n(\theta-\varphi-k z)] \\
B_{\theta}(r, \theta, z)=\frac{\mu_{0} I}{2 \pi r}+\frac{\mu_{0} I}{\pi} k b \sum_{n=1}^{\infty} n I_{n}^{\prime}(n k b) \frac{K_{n}(n k r)}{r} \cos [n(\theta-\varphi-k z)] \\
B_{z}(r, \theta, z)=-\frac{\mu_{0} I}{\pi} k^{2} b \sum_{n=1}^{\infty} n I_{n}^{\prime}(n k b) K_{n}(n k r) \cos [n(\theta-\varphi-k z)]
\end{array}\right.
\end{aligned}
$$

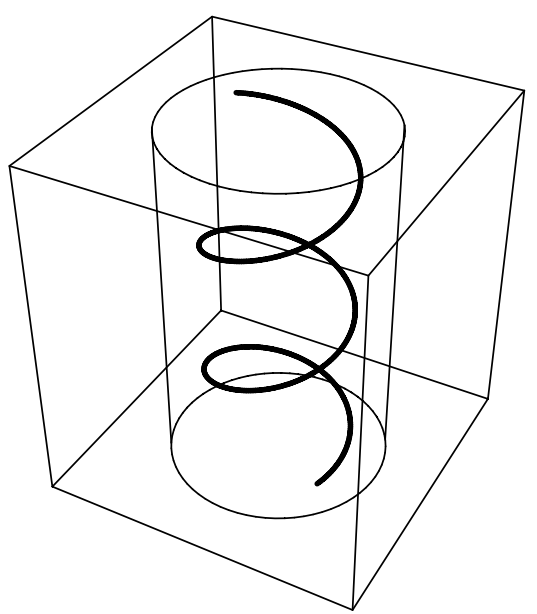

Fig. 1. Schematic view of a single helical coil placed inside a cylindrical hole in iron. 


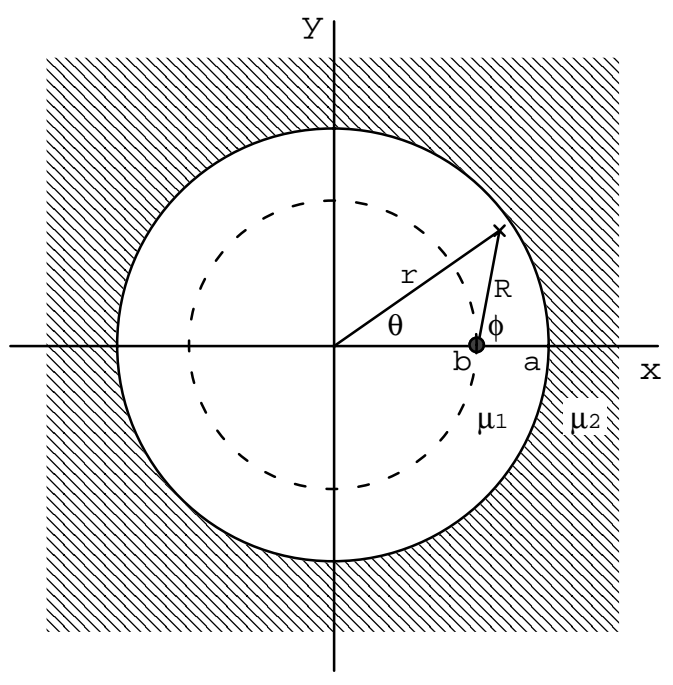

Fig. 2. Cross section of a single helical coil placed inside a cylindrical hole in iron $(\mathrm{z}=0)$.

\section{3-Dimensional POTENTIAL PROBLEM OF HELICAL MAgNETS}

On the case that a single helical current conductor with a pitch length $L(=2 \pi / \mathrm{k})$ is located at some point $(\mathrm{r}=\mathrm{b}, \theta=0)$ at the $\mathrm{z}=0$ plane placed inside a cylindrical hole in iron of $\mu_{2}=\kappa_{\mathrm{m}} \mu_{0}$, as shown in Figs. 1 and 2, the general solutions at $\mathrm{z}=0$ for the magnetic scalar potential $\phi_{1}$ and $\phi_{2}$ of both regions can be written in the following forms with the unknown constants of $A_{n}, B_{0}$, and $B_{n}$,

$\phi_{1}(r, \theta, z=0)=-\frac{I}{2 \pi} \theta-\frac{I}{\pi} k b \sum_{n=1}^{\infty} I_{n}^{\prime}(n k b) K_{n}(n k r) \sin n \theta-\frac{I}{\pi} \sum_{n=1}^{\infty} A_{n} I_{n}(n k r) \sin n \theta$

$\phi_{2}(r, \theta, z=0)=-\frac{I}{2 \pi} B_{0} \theta-\frac{I}{\pi} \sum_{n=1}^{\infty} B_{n} K_{n}(n k r) \sin n \theta$

The constants may be determined by the use of the boundary conditions on the interface, $r$ $=\mathrm{a}$, between regions 1 and 2 . On the boundary between the region $1\left(\mu_{1}=\mu_{0}\right)$ and the region $2\left(\mu_{2}=\kappa_{\mathrm{m}} \mu_{0}\right)$, the following conditions must be fulfilled,

$\left\{\begin{array}{l}H_{t 1}=H_{t 2} \\ B_{n 1}=B_{n 2}\end{array}\right.$

The continuity of the tangential component of $\mathrm{H}$ at the boundary $\mathrm{r}=\mathrm{a}$ is equivalent to the continuity of $\phi_{\mathrm{m}}$, while the continuity of the normal component of B demands that 
$\mu_{1} \frac{\partial \phi_{1}}{\partial r}=\mu_{2} \frac{\partial \phi_{2}}{\partial r}$

at $r=a$. Then, the above boundary conditions are equivalent with the following condition for the magnetic scalar potential.

$\left\{\begin{array}{l}\phi_{1}=\phi_{2} \\ \mu_{1} \frac{\partial \phi_{1}}{\partial r}=\mu_{2} \frac{\partial \phi_{2}}{\partial r}\end{array}\right.$

On the other hand, it seems wrong that the boundary condition on the inner surface of iron by Caspi is $\phi_{\text {coil }}+\phi_{\text {iron }}=\phi_{1}=$ constant at $\mathrm{r}=\mathrm{a}$.

From the first condition of Eq. (10), the following relations are obtained,

$\left\{\begin{array}{l}k b I_{n}^{\prime}(n k b) K_{n}(n k a)+A_{n} I_{n}(n k a)=B_{n} K_{n}(n k a) \\ B_{0}=1\end{array}\right.$

Similarly, from the second condition of Eq. (10), the following relation is obtained,

$k b I_{n}^{\prime}(n k b) K_{n}^{\prime}(n k a)+A_{n} I_{n}^{\prime}(n k a)=\kappa_{m} B_{n} K_{n}^{\prime}(n k a)$

As a result, the unknown constants are determined as follows,

$$
\begin{aligned}
& A_{n}=-k b \frac{\left(\kappa_{m}-1\right) K_{n}(n k a) K_{n}^{\prime}(n k a)}{\kappa_{m} I_{n}(n k a) K_{n}^{\prime}(n k a)-I_{n}^{\prime}(n k a) K_{n}(n k a)} I_{n}^{\prime}(n k b) \\
& B_{n}=-k b \frac{I_{n}^{\prime}(n k a) K_{n}(n k a)-I_{n}(n k a) K_{n}^{\prime}(n k a)}{\kappa_{m} I_{n}(n k a) K_{n}^{\prime}(n k a)-I_{n}^{\prime}(n k a) K_{n}(n k a)} I_{n}^{\prime}(n k b) \\
& =-\frac{b}{n a} \frac{1}{\kappa_{m} I_{n}(n k a) K_{n}^{\prime}(n k a)-I_{n}^{\prime}(n k a) K_{n}(n k a)} I_{n}^{\prime}(n k b)
\end{aligned}
$$

Therefore, the magnetic scalar potential $\phi_{1 \mathrm{i}}$ at $\mathrm{z}=0$ due to the contribution of an axially symmetric iron yoke on the region 1 is expressed as follows, 


$$
\begin{aligned}
& \phi_{1 i}=\frac{I}{\pi} \sum_{n=1}^{\infty} \frac{\left(\kappa_{m}-1\right) K_{n}(n k a) K_{n}^{\prime}(n k a)}{\kappa_{m} I_{n}(n k a) K_{n}^{\prime}(n k a)-I_{n}^{\prime}(n k a) K_{n}(n k a)} k b I_{n}^{\prime}(n k b) I_{n}(n k r) \sin n \theta \\
& =\frac{I}{\pi} \sum_{n=1}^{\infty} \frac{\kappa_{m}-1}{\kappa_{m}-I_{n}^{\prime}(n k a) K_{n}(n k a) / I_{n}(n k a) K_{n}^{\prime}(n k a)} \frac{K_{n}(n k a)}{I_{n}(n k a)} k b I_{n}^{\prime}(n k b) I_{n}(n k r) \sin n \theta
\end{aligned}
$$

The asymptotic forms for the following terms with the modified Bessel functions and their derivatives as $\mathrm{k} \rightarrow 0(\mathrm{~L} \rightarrow \infty)$ are as follows, [3]

$$
\begin{aligned}
& \frac{I_{n}^{\prime}(n k a) K_{n}(n k a)}{I_{n}(n k a) K_{n}^{\prime}(n k a)} \approx-1 \\
& \frac{K_{n}(n k a)}{I_{n}(n k a)} k b I_{n}^{\prime}(n k b) I_{n}(n k r) \approx \frac{1}{2 n}\left(\frac{r}{a^{2} / b}\right)^{n}
\end{aligned}
$$

Then, it can be revealed that the magnetic scalar potential $\phi_{1 \mathrm{i}}$ results the potential due to the straight image current, as $\mathrm{k} \rightarrow 0(\mathrm{~L} \rightarrow \infty)$. [4,5,6] Similarly, the magnetic scalar potential $\phi_{2}$ on the region 2 is written as the following form,

$$
\begin{aligned}
& \phi_{2}=-\frac{I}{2 \pi} \theta+\frac{I}{\pi} \sum_{n=1}^{\infty} \frac{1}{\kappa_{m} I_{n}(n k a) K_{n}^{\prime}(n k a)-I_{n}^{\prime}(n k a) K_{n}(n k a)} \frac{b}{n a} I_{n}^{\prime}(n k b) K_{n}(n k r) \sin n \theta \\
& =-\frac{I}{2 \pi} \theta+\frac{I}{\pi} \sum_{n=1}^{\infty} \frac{1}{\kappa_{m}-I_{n}^{\prime}(n k a) K_{n}(n k a) / I_{n}(n k a) K_{n}^{\prime}(n k a)} \frac{b}{n a} \frac{I_{n}^{\prime}(n k b)}{I_{n}(n k a) K_{n}^{\prime}(n k a)} K_{n}(n k r) \sin n \theta
\end{aligned}
$$

The asymptotic form for the following term as $\mathrm{k} \rightarrow 0(\mathrm{~L} \rightarrow \infty)$ are as follows, [3]

$$
\frac{b}{n a} \frac{I_{n}^{\prime}(n k b)}{I_{n}(n k a) K_{n}^{\prime}(n k a)} K_{n}(n k r) \approx-\frac{1}{n}\left(\frac{b}{r}\right)^{n}
$$

Then, it can also be revealed that the magnetic scalar potential $\phi_{2}$ results the potential of the 2-dimensional case, as $\mathrm{k} \rightarrow 0(\mathrm{~L} \rightarrow \infty)$. [4,5,6]

Finally, on the case that a single helical current carrying conductor with a pitch length $\mathrm{L}$ $(=2 \pi / \mathrm{k})$ is located at some point $(\mathrm{r}=\mathrm{b}, \theta=\varphi)$ at the $\mathrm{z}=0$ plane, the general form for the 
magnetic scalar potential $\phi_{1 \mathrm{i}}$ due to an axially symmetric iron yoke on the region 1 is expressed as follows,

$$
\phi_{1 i}(r, \theta, z)=\frac{I}{\pi} \sum_{n=1}^{\infty}\left\{\frac{\kappa_{m}-1}{\kappa_{m}-I_{n}^{\prime}(n k a) K_{n}(n k a) / I_{n}(n k a) K_{n}^{\prime}(n k a)}\right\} \frac{K_{n}(n k a)}{I_{n}(n k a)} k b I_{n}^{\prime}(n k b) I_{n}(n k r) \sin [n(\theta-\varphi-k z)]
$$

The field B can be calculated from the following equation,

$$
\left\{\begin{array}{l}
B_{1}=-\mu_{1} \nabla \phi_{1}=-\mu_{0} \nabla \phi_{1} \\
B_{2}=-\mu_{2} \nabla \phi_{2}=-\kappa_{m} \mu_{0} \nabla \phi_{2}
\end{array}\right.
$$

As a result, the field $B_{i}$ due to the contribution of an axially symmetric iron yoke on the region 1 is obtained as follows,

$$
\left\{\begin{array}{l}
B_{r i}(r, \theta, z)= \\
-\frac{\mu_{0} I}{\pi} k^{2} b \sum_{n=1}^{\infty} n\left\{\frac{\kappa_{m}-1}{\kappa_{m}-I_{n}^{\prime}(n k a) K_{n}(n k a) / I_{n}(n k a) K_{n}^{\prime}(n k a)}\right\} \frac{K_{n}(n k a)}{I_{n}(n k a)} I_{n}^{\prime}(n k b) I_{n}^{\prime}(n k r) \sin [n(\theta-\varphi-k z)] \\
B_{\theta i}(r, \theta, z)= \\
-\frac{\mu_{0} I}{\pi} k b \sum_{n=1}^{\infty} n\left\{\frac{\kappa_{m}-1}{\kappa_{m}-I_{n}^{\prime}(n k a) K_{n}(n k a) / I_{n}(n k a) K_{n}^{\prime}(n k a)}\right\} \frac{K_{n}(n k a)}{I_{n}(n k a)} I_{n}^{\prime}(n k b) \frac{I_{n}(n k r)}{r} \cos [n(\theta-\varphi-k z)] \\
B_{z i}(r, \theta, z)= \\
+\frac{\mu_{0} I}{\pi} k^{2} b \sum_{n=1}^{\infty} n\left\{\frac{\kappa_{m}-1}{\kappa_{m}-I_{n}^{\prime}(n k a) K_{n}(n k a) / I_{n}(n k a) K_{n}^{\prime}(n k a)}\right\} \frac{K_{n}(n k a)}{I_{n}(n k a)} I_{n}^{\prime}(n k b) I_{n}(n k r) \cos [n(\theta-\varphi-k z)]
\end{array}\right.
$$

\section{FIELD CALCULATION FOR HELICAL DIPOLE PROTOTYPES}

\subsection{Prototype with the Half-length}

The main parameters of the slotted helical dipole prototype with the half-length for RHIC are listed in Table 1 of reference [2]. The cross section of the slotted helical dipole prototype with the half-length for RHIC is shown in Fig. 12 of reference [2].

The analytically and numerically calculated and measured results of the multipoles for a single current of 200 A are listed in Table 1, together with the newly calculated results. Table 1 is revised from Table 2 of reference [2]. The measured data is the results by the rotating coil of the tangential winding. On the previous calculation, the contribution of an axially symmetric iron yoke or the effect of the iron yoke is approximately calculated with 
the simple assumption that the helical image current is the same with the case of the straight current for the position (or radius) and the intensity of the image current. This calculated results are referred as the old analytical calculation (old analytical) in Table 1. The contribution of an axially symmetric iron yoke is newly calculated, using Eq. (22), based on the rigorous treatment of the potential problem, while the contribution of the helical coil is calculated, using Eq. (4) similarly with the previous calculation. The agreement with the numerical calculation by TOSCA and the measured result is greatly improved, as shown in Table 1. This newly calculated results are referred as the new analytical calculation (new analytical) in Table 1. In this new calculation, the relative permeability of iron yoke is also assumed to be infinite, and the length of the magnet is also infinite. The helical dipole reference field $\tilde{B}_{\text {ref }}$, the helical normal and skew multipole coefficients $\tilde{b}_{n}$ and $\tilde{a}_{n}$ are defined by the following equations,

$$
\left\{\begin{array}{l}
B_{r}(r, \theta, z)=\tilde{B}_{r e f} r_{0} \sum_{n=1}^{\infty} n !\left(\frac{2}{n k r_{0}}\right)^{n} k I_{n}^{\prime}(n k r)\left\{-\tilde{a}_{n} \cos [n(\theta-k z)]+\tilde{b}_{n} \sin [n(\theta-k z)]\right\} \\
B_{\theta}(r, \theta, z)=\tilde{B}_{r e f} r_{0} \sum_{n=1}^{\infty} n !\left(\frac{2}{n k r_{0}}\right)^{n} \frac{I_{n}(n k r)}{r}\left\{\tilde{a}_{n} \sin [n(\theta-k z)]+\tilde{b}_{n} \cos [n(\theta-k z)]\right\} \\
B_{z}(r, \theta, z)=\tilde{B}_{r e f} r_{0} \sum_{n=1}^{\infty}(-k) n !\left(\frac{2}{n k r_{0}}\right)^{n} I_{n}(n k r)\left\{\tilde{a}_{n} \sin [n(\theta-k z)]+\tilde{b}_{n} \cos [n(\theta-k z)]\right\}
\end{array}\right.
$$

where $r_{0}$ is the reference radius of $31 \mathrm{~mm} . \quad \tilde{B}_{r e f, c o i l}$ is the contribution due to the helical coil for the helical dipole reference field.

The comparison among the new analytical calculation with the linear current dependence (solid), the old analytical (dashing), and measured results by rotating coils (line with dots) for the current dependence of the helical dipole reference field $\tilde{B}_{r e f}$ is shown in Fig. 3 . The measured data contains the results by three rotating coils, dipole \#1, dipole \#2, and tangential windings. The difference among the results of the helical dipole reference field $\tilde{B}_{\text {ref }}$ by three rotating coils is not large, as shown in Fig. 3. Contour plot of the vertical field component, $\mathrm{B}_{\mathrm{y}}$, derived (or synthesized) from the new analytical calculation up to 18-pole at $\mathrm{I}=200$ A listed in Table 1, is revised, as shown in Fig. 4, instead of Fig. 13 of reference [2]. The difference between both the new and old contour plots looks quite small, resulting from the small difference between 2 calculated results for the helical normal multipole coefficients $\tilde{b}_{n}$. 
Table 1. Helical multipole coefficients $\left(10^{-4}\right)$ for the half-length helical dipole prototype, at $\mathrm{I}=200 \mathrm{~A}$.

\begin{tabular}{|c|c|c|c|c|c|c|}
\hline $\mathrm{n}$ & Pole & $\begin{array}{c}\tilde{b}_{n} \\
\text { (old analytical) }\end{array}$ & $\begin{array}{c}\tilde{b}_{n} \\
\text { (new analytical) }\end{array}$ & $\begin{array}{c}\tilde{b}_{n} \\
\text { (TOSCA) }\end{array}$ & $\tilde{b}_{n}$ & red) \\
\hline$\tilde{B}_{r e f}(\mathrm{~T})$ & & 2.81 & 2.72 & 2.71 & \multicolumn{2}{|c|}{2.72} \\
\hline$\tilde{B}_{r e f, c o i l}(\mathrm{~T})$ & & 1.75 & 1.75 & - & \multicolumn{2}{|c|}{-} \\
\hline 2 & quadrupole & & & & 1.2 & -0.41 \\
\hline 3 & sextupole & -49.3 & -50.8 & -52.4 & -63.2 & -0.26 \\
\hline 4 & octupole & & & & 2.3 & 0.03 \\
\hline 5 & decapole & 5.5 & 5.7 & 6.0 & 9.7 & 2.4 \\
\hline 6 & dodecapole & & & & -0.54 & -1.2 \\
\hline 7 & 14 - pole & 0.29 & 0.29 & 0.29 & 3.3 & 7.3 \\
\hline 8 & 16 - pole & & & & 0.51 & -4.0 \\
\hline 9 & 18 - pole & -7.6 & -7.8 & -7.8 & -19.7 & 1.6 \\
\hline 10 & 20 - pole & & & & 20.5 & 6.9 \\
\hline
\end{tabular}

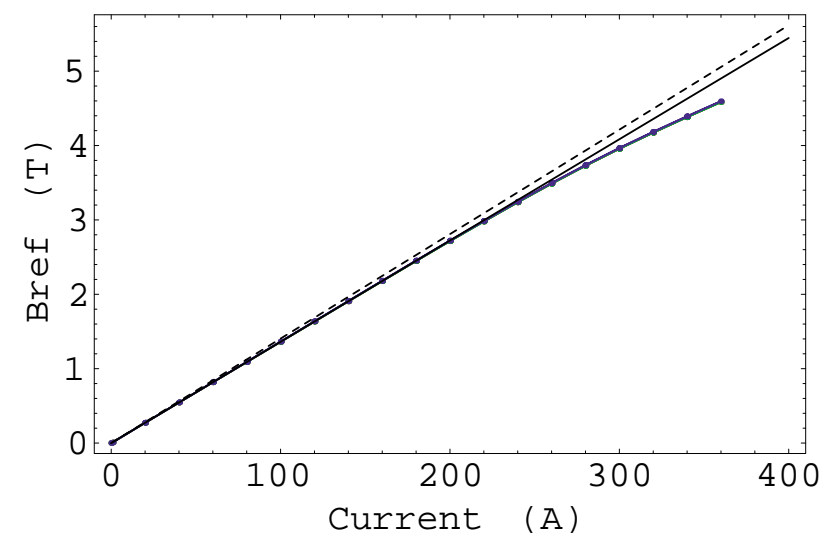

Fig. 3. Comparison among the new analytical linear calculation (solid), the old analytical (dashing), and measured results by rotating coils (line with dots) for the current dependence of the helical dipole reference field $\tilde{B}_{r e f}$. 


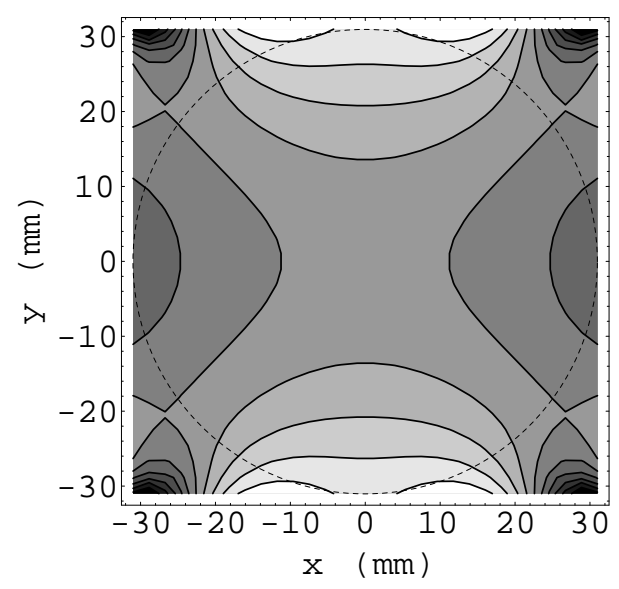

Fig. 4. Contour plot of $\mathrm{B}_{\mathrm{y}}$ at $\mathrm{I}=200 \mathrm{~A}$ (new analytical calculation).

\subsection{Prototype with the Full-length}

The magnetic field of the slotted helical dipole prototype with the full-length for RHIC is also analytically recalculated, using Eq. (22) for the contribution of an axially symmetric iron yoke. The cross section of the slotted helical dipole prototype with the full-length for RHIC is shown in Fig. 5 of reference [7]. Contour plot of the vertical field component, $\mathrm{B}_{\mathrm{y}}$, derived (or synthesized) from the new analytical calculation up to 18-pole at $\mathrm{I}=300 \mathrm{~A}$ listed in Table 2, is revised, as shown in Fig. 5 instead of Fig. 6 of reference [7]. The difference between both the new and old contour plots looks also quite small.

Table 2. Helical multipole coefficients $\left(10^{-4}\right)$ for the full-length helical dipole.

\begin{tabular}{cccccc}
\hline $\mathrm{n}$ & Pole & $\begin{array}{c}\tilde{b}_{n} \\
(\text { old analytical) }\end{array}$ & $\begin{array}{c}\tilde{b}_{n} \\
\text { (new analytical) }\end{array}$ & $\begin{array}{c}\tilde{b}_{n} \\
\text { (TOSCA) }\end{array}$ & $\begin{array}{c}\tilde{b}_{n} \\
\text { (TOSCA) }\end{array}$ \\
\hline Current (A) & & $300(87)$ & $300(87)$ & 300 & 87 \\
$\tilde{B}_{\text {ref }}(\mathrm{T})$ & & $4.15(1.20)$ & $4.03(1.17)$ & 3.88 & 1.19 \\
$\tilde{B}_{\text {ref,coil }}(\mathrm{T})$ & & $2.60(0.754)$ & $2.60(0.754)$ & - & - \\
3 & sextupole & 6.2 & 6.2 & 3.7 & 6.0 \\
5 & decapole & -0.34 & -0.34 & -2.6 & 0.40 \\
7 & $14-$ pole & -0.84 & -0.86 & -1.0 & -0.32 \\
9 & $18-$ pole & -7.4 & -7.6 & -8.1 & -7.1 \\
\hline
\end{tabular}




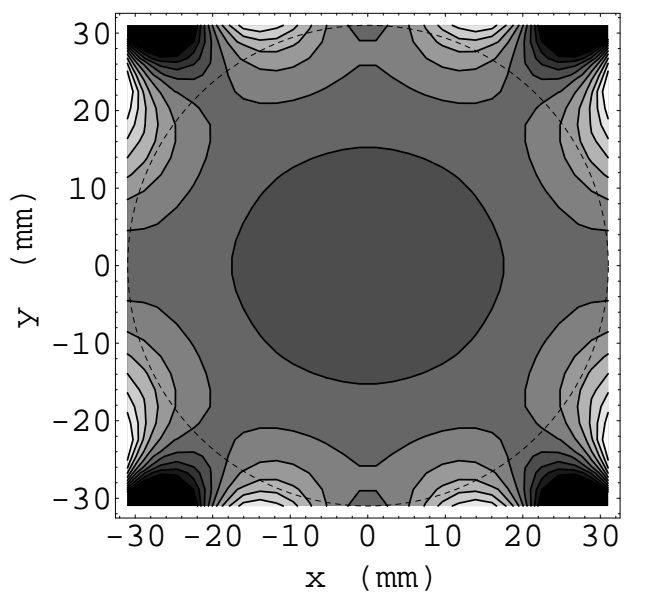

Fig. 5. Contour plot of $\mathrm{B}_{\mathrm{y}}$ at $\mathrm{I}=300 \mathrm{~A}$ (new analytical calculation).

\section{CONCLUSION}

For the analytical field calculation for the helical magnets with an axially symmetric iron yoke, the 3-dimensoinal potential problem for the case that a helical current carrying conductor is placed inside a cylindrical hole in iron is solved. The obtained results are applied for the slotted helical dipole prototype with the half-length and full-length for RHIC, with good agreement among the other numerical calculations and measured results.

\section{ACKNOWLEDGMENTS}

The authors are indebted for helpful discussions and comments to the BNL/RIKEN RHIC spin accelerator group.

\section{References}

[1] S. Caspi, "Magnetic Field Components in a Sinusoidally Varying Helical Wiggler", SC-MAG-464, LBL-35928, (1994).

[2] T. Tominaka, M. Okamura, and T. Katayama, "Analytical Field Calculation of Helical Coils", Nucl. Instrum. \& Methods in Physics Research (A), 459 (2001) pp.398-411.

[3] M. Abramowitz and I. A. Stegun (eds.), "Handbook of Mathematical Functions", Dover, pp.374-376 (1970).

[4] B. Hague, "The Principles of Electromagnetism Applied to Electrical Machines", Dover, p.124 (1962).

[5] M. Stafl, "Electrodynamics of Electrical Machines", Academia, p.84 (1967).

[6] W. R. Smythe, "Static and Dynamic Electricity", McGraw-Hill, p.310 (1968).

[7] T. Tominaka, M. Okamura, and T. Katayama, "Field Distributions of the Slotted Helical Dipole Prototypes with the Half and Full-Length", AGS/RHIC/SN No.74, July 22, (1998). 